\title{
Global Analysis of a Model of Viral Infection with Latent Stage and Two Types of Target Cells
}

\author{
Shuo Liu, ${ }^{1}$ Lina $\mathrm{Ma},{ }^{2}$ Jianquan $\mathrm{Li}^{3}$ and Qingbo $\mathrm{Zhao}^{1}$ \\ ${ }^{1}$ School of Biomedical Engineering, The Fourth Military Medical University, Xian 710032, China \\ ${ }^{2}$ College of Mathematics and Information Science, Shaanxi Normal University, Xian 710062, China \\ ${ }^{3}$ College of Science, Air Force Engineering University, Xian 710051, China \\ Correspondence should be addressed to Jianquan Li; jianq_li@263.net
}

Received 23 June 2013; Accepted 8 September 2013

Academic Editor: Fernando Simões

Copyright (C) 2013 Shuo Liu et al. This is an open access article distributed under the Creative Commons Attribution License, which permits unrestricted use, distribution, and reproduction in any medium, provided the original work is properly cited.

\begin{abstract}
By introducing the probability function describing latency of infected cells, we unify some models of viral infection with latent stage. For the case that the probability function is a step function, which implies that the latency period of the infected cells is constant, the corresponding model is a delay differential system. The model with delay of latency and two types of target cells is investigated, and the obtained results show that when the basic reproduction number is less than or equal to unity, the infectionfree equilibrium is globally stable, that is, the in-host free virus will be cleared out finally; when the basic reproduction number is greater than unity, the infection equilibrium is globally stable, that is, the viral infection will be chronic and persist in-host. And by comparing the basic reproduction numbers of ordinary differential system and the associated delayed differential system, we think that it is necessary to elect an appropriate type of probability function for predicting the final outcome of viral infection in-host.
\end{abstract}

\section{Introduction}

The dynamical models of virus infection have played an important role in understanding the action of in-host free virus on target cells. Nowak et al. [1,2] proposed one of the earliest of these models:

$$
\begin{gathered}
x^{\prime}=\lambda-d x-\beta x v, \\
y^{\prime}=\beta x v-a y, \\
v^{\prime}=k a y-\gamma v,
\end{gathered}
$$

where $x=x(t), y=y(t)$, and $v=v(t)$ are the concentrations of uninfected cells, infected cells, and viral particles (virions) at time $t$, respectively. In model (1), uninfected target cells are assumed to be produced at a constant rate $\lambda$ and die at a rate $d x$. Infection of target cells by in-host free virus is assumed to occur at a bilinear rate $\beta x v$. Infected cells are lost at a rate ay. Free virus are produced by infected cells at a rate kay in which $k$ is the average number of viral particles produced by a single infected cell over its lifetime and die at a rate $\gamma \nu$.
Model (1) is a basic model of viral infection, which has been used widely to investigate infection of some viruses (such as, HIV, HBV, HCV, and HLTV). However, following infection of virus, within a cell the provirus may remain latent, giving no sign of its presence for months or years [3]. According to this fact, in order to investigate HIV-1 dynamics in vivo, Perelson et al. incorporated the latently infected cells into the basic model $(1)[4,5]$. It implies that the development of infected cell should include latent and active two stages. That is, once infected, a cell first becomes a latently infected cell, but does not produce virus; after a period of time a latently infected cell turns active and begins to produce virus. The global stability of some ODE models of viral infection with latent stage is considered in $[6,7]$.

In 1997, Perelson et al. [8] observed that HIV attacks two types of target cells, $\mathrm{CD} 4^{+} \mathrm{T}$ cells and macrophages. On the other hand, it was also detected that, except for liver tissue, HCV may be produced in some extrahepatic tissues, such as bone marrow [9], peripheral blood mononuclear cells (PBMC) [10], brain [11], and lymph nodes [12]. Then, according to these virological findings, based on model (1), 
some viral dynamical models with two types of target cells were proposed $[5,13,14]$, which are all expressed by ordinary differential equations.

Since the dynamics of viral infection in-host is not well understood, in order to investigate the mechanism of viral infection, some reasonable assumptions are often incorporated into mathematical models describing the interaction between target cells and viral particles. In this paper, we first introduce the probability functions describing the latency of infected cells to unify some models of viral infection with latent stage and then analyze dynamics of viral infection model with constant latency period and two types of target cells. The discussed model is a system of delay differential equations.

The organization of this paper is as follows. In Section 2, we unify some models of viral infection with latent stage by introducing the probability function describing latency of infected cells and propose a model of virus infection with latent delay and two types of target cells. The global stability is analyzed in Section 3. At last, the conclusion on the model is summarized, and the basic reproduction numbers of ordinary differential system and the associated delay differential system are compared.

\section{Models}

Let $Q(t)$ for $t \geq 0$ denote the probability that an infected cell is in the latent stage at least $t$ time units before becoming the actively infected cell, and then, when the infection rate of virus is assumed to be $\beta x(t) v(t)$, the concentration of the latently infected cells at time $t$ can be expressed by the following equation:

$$
w(t)=w_{0}(t)+\int_{0}^{t} \beta x(\theta) v(\theta) Q(t-\theta) e^{-d(t-\theta)} d \theta
$$

where $w_{0}(t)$ is the concentration of the latently infected cells which are in the latent stage at time 0 and still at the same state at time $t$. Function $w_{0}(t)$ is a nonnegative, nonincreasing, and piecewise continuous function. Thus, when incorporating the latent stage of infected cells into the basic model (1), we have the model of viral infection with latent stage:

$$
\begin{gathered}
x^{\prime}=\lambda-d x-\beta x v, \\
w(t)=w_{0}(t)+\int_{0}^{t} \beta x(\theta) v(\theta) Q(t-\theta) e^{-d(t-\theta)} d \theta, \\
y^{\prime}=F(x, y, w)-a y, \\
v^{\prime}=k a y-\gamma v,
\end{gathered}
$$

where the term $F(x, y, w)$ in the third equation of (3) represents the recruitment rate of actively infected cells; its expression should depend on the form of function $Q(t)$.

Usually, function $Q(t)$ is elected as one of the following two types, the exponential function (i.e., $Q(t)=\exp (-\varepsilon t)$ ) and the step function (i.e., $Q(t)$ equals to 1 for $t \in[0, \tau)$ and 0 for $t \in[\tau,+\infty))[15,16]$. Here, the exponential function means that the transfer of the infected cells from the latent state to the active one follows the exponential distribution, and the step function means that the time length of staying at the latent state for the infected cells is constant $\tau$ and that they become active after the time period $\tau$. It is easy to know that the average latency period of the infected cells is $1 / \varepsilon$ for the exponential function and $\tau$ for the step function.

When $Q(t)=\exp (-\varepsilon t),(2)$ becomes

$$
w(t)=w_{0} e^{-(d+\varepsilon) t}+\int_{0}^{t} \beta x(\theta) v(\theta) e^{-(d+\varepsilon)(t-\theta)} d \theta
$$

where $w_{0}$ is the concentration of the latently infected cells at $t=0$, then we have

$$
w^{\prime}(t)=\beta x(t) v(t)-(d+\varepsilon) w(t) .
$$

From the equation of $w$, we know that the removed rate of the latently infected cells is $(d+\varepsilon) w$, where $d$ is the death rate coefficient of uninfected cells; then $\varepsilon w$ is the infection-induced transfer rate to actively infected cells, that is, $F(x, y, w)=\varepsilon w$. So, system (3) becomes

$$
\begin{gathered}
x^{\prime}=\lambda-d x-\beta x v, \\
w^{\prime}=\beta x v-(d+\varepsilon) w, \\
y^{\prime}=\varepsilon w-a y, \\
v^{\prime}=k a y-\gamma v .
\end{gathered}
$$

When $Q(t)$ is a step function, that is,

$$
Q(t)= \begin{cases}1 & \text { for } t \in[0, \tau), \\ 0 & \text { for } t \in[\tau,+\infty),\end{cases}
$$

$w_{0}(t)=0$ for $t \geq \tau$. Thus, for $t \geq \tau$ the integral equation (2) becomes

$$
w(t)=\int_{t-\tau}^{t} \beta x(\theta) v(\theta) e^{-d(t-\theta)} d \theta
$$

It is equivalent to the following delay differential equation:

$$
w^{\prime}(t)=\beta x(t) v(t)-\beta e^{-d \tau} x(t-\tau) v(t-\tau)-d w(t),
$$

with $w(0)=\int_{-\tau}^{0} \beta x(\theta) v(\theta) e^{d \theta} d \theta$, where the term $\beta e^{-d \tau} x(t-\tau)$ $v(t-\tau)$ represents the recruitment rate of the actively infected cells for $t>\tau$. Thus, when investigating the longterm behavior of model (3), the corresponding model with constant latent period is given by

$$
\begin{gathered}
x^{\prime}=\lambda-d x-\beta x v, \\
w^{\prime}=\beta x v-\beta e^{-d \tau} x(t-\tau) v(t-\tau)-d w, \\
y^{\prime}=\beta e^{-d \tau} x(t-\tau) v(t-\tau)-a y, \\
v^{\prime}=k a y-\gamma v .
\end{gathered}
$$

From the inference above, we may see that models (6) and (10) can be unified into model (3) and are two special cases 
of (3). This is due to the introduction of the probability function $Q(t)$. Global properties of models (6) and (10) were investigated in $[6,17]$, respectively.

When considering the case that virus attacks two types of target cells, we denote the corresponding quantities by the same letters as model (3) with the subscript 1 or 2 . The subscript represents the type of target cells. Thus, we have the following model with latent stage and two types of target cells:

$$
\begin{gathered}
x_{1}^{\prime}=\lambda_{1}-d_{1} x_{1}-\beta_{1} x_{1} v, \\
w_{1}(t)=w_{10}(t) \\
+\int_{0}^{t} \beta_{1} x_{1}(\theta) v(\theta) Q_{1}(t-\theta) e^{-d_{1}(t-\theta)} d \theta, \\
y_{1}^{\prime}=F_{1}\left(x_{1}, y_{1}, w_{1}\right)-a_{1} y_{1}, \\
x_{2}^{\prime}=\lambda_{2}-d_{2} x_{2}-\beta_{2} x_{2} v, \\
w_{2}(t)=w_{20}(t) \\
+\int_{0}^{t} \beta_{2} x_{2}(\theta) v(\theta) Q_{2}(t-\theta) e^{-d_{2}(t-\theta)} d \theta, \\
y_{2}^{\prime}=F_{2}\left(x_{2}, y_{2}, w_{2}\right)-a_{2} y_{2}, \\
v^{\prime}=k_{1} a_{1} y_{1}+k_{2} a_{2} y_{2}-\gamma v .
\end{gathered}
$$

Similarly, when the probability functions in (11) are exponential function, model (11) can become

$$
\begin{gathered}
x_{1}^{\prime}=\lambda_{1}-d_{1} x_{1}-\beta_{1} x_{1} v, \\
w_{1}^{\prime}=\beta_{1} x_{1} v-\left(d_{1}+\varepsilon_{1}\right) w_{1}, \\
y_{1}^{\prime}=\varepsilon_{1} w_{1}-a_{1} y_{1}, \\
x_{2}^{\prime}=\lambda_{2}-d_{2} x_{2}-\beta_{2} x_{2} v, \\
w_{2}^{\prime}=\beta_{2} x_{2} v-\left(d_{2}+\varepsilon_{2}\right) w_{2}, \\
y_{2}^{\prime}=\varepsilon_{2} w_{2}-a_{2} y_{2}, \\
v^{\prime}=k_{1} a_{1} y_{1}+k_{2} a_{2} y_{2}-\gamma v .
\end{gathered}
$$

Its dynamical behavior was analyzed in [7].

When the probability functions in (11) are step function, for $t>\tau:=\max \left\{\tau_{1}, \tau_{2}\right\}$, model (11) can become

$$
\begin{gathered}
x_{1}^{\prime}(t)=\lambda_{1}-d_{1} x_{1}(t)-\beta_{1} x_{1}(t) v(t), \\
w_{1}^{\prime}(t)=\beta_{1} x_{1}(t) v(t)-\beta_{1} e^{-d_{1} \tau_{1}} x_{1}\left(t-\tau_{1}\right) \\
\quad \times v\left(t-\tau_{1}\right)-d_{1} w_{1}(t), \\
y_{1}^{\prime}(t)=\beta_{1} e^{-d_{1} \tau_{1}} x_{1}\left(t-\tau_{1}\right) v\left(t-\tau_{1}\right)-a_{1} y_{1}(t),
\end{gathered}
$$

$$
\begin{gathered}
x_{2}^{\prime}(t)=\lambda_{2}-d_{2} x_{2}(t)-\beta_{2} x_{2}(t) v(t), \\
w_{2}^{\prime}(t)=\beta_{2} x_{2}(t) v(t)-\beta_{2} e^{-d_{2} \tau_{2}} x_{2}(t-\tau) \\
\times v\left(t-\tau_{2}\right)-d_{2} w_{2}(t), \\
y_{2}^{\prime}=\beta_{2} e^{-d_{2} \tau_{2}} x_{2}\left(t-\tau_{2}\right) v\left(t-\tau_{2}\right)-a_{2} y_{2}(t), \\
v^{\prime}=k_{1} a_{1} y_{1}(t)+k_{2} a_{2} y_{2}(t)-\gamma v(t) .
\end{gathered}
$$

For system (13), variables $w_{1}$ and $w_{2}$ do not appear in the equations of $x_{i}, y_{i}(i=1,2)$, and $v$, then denoting $b_{1}=e^{-d_{1} \tau_{1}}$, and $b_{2}=e^{-d_{2} \tau_{2}}$, gives a subsystem of (13) as follows:

$$
\begin{gathered}
x_{1}^{\prime}(t)=\lambda_{1}-d_{1} x_{1}(t)-\beta_{1} x_{1}(t) v(t), \\
y_{1}^{\prime}(t)=\beta_{1} b_{1} x_{1}\left(t-\tau_{1}\right) v\left(t-\tau_{1}\right)-a_{1} y_{1}(t), \\
x_{2}^{\prime}(t)=\lambda_{2}-d_{2} x_{2}(t)-\beta_{2} x_{2}(t) v(t), \\
y_{2}^{\prime}=\beta_{2} b_{2} x_{2}\left(t-\tau_{2}\right) v\left(t-\tau_{2}\right)-a_{2} y_{2}(t), \\
v^{\prime}=k_{1} a_{1} y_{1}(t)+k_{2} a_{2} y_{2}(t)-\gamma v(t) .
\end{gathered}
$$

In this paper, we will investigate the global behaviors of system (14).

For system (14), we set a suitable phase space. Denote the Banach space of continuous functions mapping the interval $[-\tau, 0]$ into $\mathbb{R}^{5}$ with the sup-norm for $\phi=\left(\phi_{1}, \phi_{2}\right.$, $\left.\phi_{3}, \phi_{4}, \phi_{5}\right)^{T} \in C$ by $C=C\left([-\tau, 0], \mathbb{R}^{5}\right)$, where $\tau=\max \left\{\tau_{1}, \tau_{2}\right\}$. The nonnegative cone of $C$ is defined as $C_{+}=C\left([-\tau, 0] ; \mathbb{R}_{+}^{5}\right)$. From the biological meaning, the initial conditions for system (14) are given as follows:

$$
\begin{gathered}
x_{1}(\theta)=\phi_{1}(\theta), \quad y_{1}(\theta)=\phi_{2}(\theta), \quad x_{2}(\theta)=\phi_{3}(\theta), \\
y_{2}(\theta)=\phi_{4}(\theta), \quad v(\theta)=\phi_{5}(\theta), \quad \theta \in[-\tau, 0],
\end{gathered}
$$

where $\left(\phi_{1}(\theta), \phi_{2}(\theta), \phi_{3}(\theta), \phi_{4}(\theta), \phi_{5}(\theta)\right)^{T} \in C_{+}$and $\phi_{i}(0)>0$, $i=1,2,3,4,5$.

Under the initial conditions (15), it is easy to see that all solutions of system (14) are positive on $[0,+\infty)$. Furthermore, we have the following statement with respect to the boundedness of solutions of system (14).

Theorem 1. All solutions of system (14) under the initial conditions (15) are ultimately bounded.

Proof. Define a Lyapunov functional $L_{01}=b_{1} x_{1}(t)+y_{1}\left(t+\tau_{1}\right)$ then from the first two equations of (14), we have

$$
\begin{aligned}
L_{01}^{\prime} & =b_{1} \lambda_{1}-\left[b_{1} d_{1} x_{1}(t)+a_{1} y_{1}\left(t+\tau_{1}\right)\right] \\
& \leq b_{1} \lambda_{1}-\rho_{1} L_{01},
\end{aligned}
$$

where $\rho_{1}=\min \left\{d_{1}, a_{1}\right\}$. It follows that $\lim _{\sup } \operatorname{si+\infty }_{t \rightarrow}\left[b_{1} x_{1}(t)+\right.$ $\left.y_{1}\left(t+\tau_{1}\right)\right] \leq b_{1} \lambda_{1} / \rho_{1}$, that is, for any positive number $\varepsilon$ there is $T_{1}>0$ such that $b_{1} x_{1}(t)+y_{1}\left(t+\tau_{1}\right)<b_{1} \lambda_{1} / \rho_{1}+\varepsilon$ for $t>T_{1}$. 
Similarly, from the third and fourth equations of (14) we know that for any positive number $\varepsilon$ there is $T_{2}>0$ such that $b_{2} x_{2}(t)+y_{2}\left(t+\tau_{2}\right)<b_{2} \lambda_{2} / \rho_{2}+\varepsilon$ for $t>T_{2}$, where $\rho_{2}=$ $\min \left\{d_{2}, a_{2}\right\}$.

Therefore, for $t>T=\max \left\{T_{1}+\tau, T_{2}+\tau\right\}$, we have $y_{1}(t)<b_{1} \lambda_{1} / \rho_{1}+\varepsilon$ and $y_{2}(t)<b_{2} \lambda_{2} / \rho_{2}+\varepsilon$. Thus, from the last equation of (14), it follows that

$$
v^{\prime}(t)<k_{1} a_{1}\left(\frac{b_{1} \lambda_{1}}{\rho_{1}}+\varepsilon\right)+k_{2} a_{2}\left(\frac{b_{2} \lambda_{2}}{\rho_{2}}+\varepsilon\right)-\gamma v(t),
$$

for $t>T$. It implies that

$$
\limsup _{t \rightarrow+\infty}(t) \leq \frac{1}{\gamma}\left[k_{1} a_{1}\left(\frac{b_{1} \lambda_{1}}{\rho_{1}}+\varepsilon\right)+k_{2} a_{2}\left(\frac{b_{2} \lambda_{2}}{\rho_{2}}+\varepsilon\right)\right] .
$$

Summarizing the above inference, Theorem 1 holds.

Since the positive number $\varepsilon$ in the proof of Theorem 1 is arbitrary, we can know that the set

$$
\begin{gathered}
\Omega=\left\{x^{T}(t) \in C_{+}: b_{i} x_{i}(t)+y_{i}\left(t+\tau_{i}\right)\right. \\
\left.\leq \frac{b_{i} \lambda_{i}}{\rho_{i}}, i=1,2, v(t) \leq \bar{\rho}\right\}
\end{gathered}
$$

is positively invariant to system (14), where $x(t)=\left(x_{1}(t)\right.$, $\left.y_{1}(t), x_{2}(t), y_{2}(t), v(t)\right), \bar{\rho}=\left(k_{1} a_{1} b_{1} \lambda_{1} / \rho_{1}+k_{2} a_{2} b_{2} \lambda_{2} / \rho_{2}\right) / \gamma$. Therefore, we will consider system (14) on the set $\Omega$.

\section{Global Stability}

In this section, we will investigate the existence and stability of equilibria of system (14).

Obviously, (14) always has the infection-free equilibrium $E_{0}\left(x_{10}, 0, x_{20}, 0,0\right)$, where $x_{10}=\lambda_{1} / d_{1}$ and $x_{20}=\lambda_{2} / d_{2}$. The infection equilibrium $E^{*}\left(x_{1}^{*}, y_{1}^{*}, x_{2}^{*}, y_{2}^{*}, v^{*}\right)\left(v^{*}>0\right)$ is determined by the following equations:

$$
\begin{gathered}
\lambda_{1}-d_{1} x_{1}-\beta_{1} x_{1} v=0, \\
\beta_{1} b_{1} x_{1} v-a_{1} y_{1}=0, \\
\lambda_{2}-d_{2} x_{2}-\beta_{2} x_{2} v=0, \\
\beta_{2} b_{2} x_{2} v-a_{2} y_{2}=0, \\
k_{1} a_{1} y_{1}+k_{2} a_{2} y_{2}-\gamma v=0 .
\end{gathered}
$$

From the first and third equations of (20), we have

$$
x_{1}=\frac{\lambda_{1}}{d_{1}+\beta_{1} v}, \quad x_{2}=\frac{\lambda_{2}}{d_{2}+\beta_{2} v},
$$

respectively. Substituting them into the second and fourth equations of (20) yields

$$
y_{1}=\frac{\beta_{1} b_{1} \lambda_{1} v}{a_{1}\left(d_{1}+\beta_{1} v\right)}, \quad y_{2}=\frac{\beta_{2} b_{2} \lambda_{2} v}{a_{2}\left(d_{2}+\beta_{2} v\right)},
$$

respectively. When $v \neq 0$, substituting the above $y_{1}$ and $y_{2}$ into the last equation of (20) gives

$$
\frac{k_{1} \beta_{1} b_{1} \lambda_{1}}{d_{1}+\beta_{1} v}+\frac{k_{2} \beta_{2} b_{2} \lambda_{2}}{d_{2}+\beta_{2} v}=\gamma \text {. }
$$

Since the function of $v$ at the left hand side of (23) is strictly decreasing, it is easy to see that (23) has a positive root if and only if $k_{1} \beta_{1} b_{1} \lambda_{1} / d_{1}+k_{2} \beta_{2} b_{2} \lambda_{2} / d_{2}>\gamma$ and that the positive root is unique, denoted by $v^{*}$. Therefore, with respect to the existence of equilibria of (14), we have the following result.

Theorem 2. Denote

$$
R_{0}=\left(\frac{k_{1} \beta_{1} b_{1} \lambda_{1}}{d_{1}}+\frac{k_{2} \beta_{2} b_{2} \lambda_{2}}{d_{2}}\right) \frac{1}{\gamma}
$$

then, when $R_{0} \leq 1$, system (14) only has the infection-free equilibrium $E_{0}\left(x_{10}, 0, x_{20}, 0,0\right)$, where $x_{10}=\lambda_{1} / d_{1}$ and $x_{20}=$ $\lambda_{2} / d_{2}$; when $R_{0}>1$, besides $E_{0}$, system (14) also has a unique infection equilibrium $E^{*}\left(x_{1}^{*}, y_{1}^{*}, x_{2}^{*}, y_{2}^{*}, v^{*}\right)$, where

$$
\begin{array}{cc}
x_{1}^{*}=\frac{\lambda_{1}}{d_{1}+\beta_{1} v^{*}}, & x_{2}^{*}=\frac{\lambda_{2}}{d_{2}+\beta_{2} v^{*}}, \\
y_{1}^{*}=\frac{\beta_{1} b_{1} \lambda_{1} v^{*}}{a_{1}\left(d_{1}+\beta_{1} v^{*}\right)}, & y_{2}^{*}=\frac{\beta_{2} b_{2} \lambda_{2} v^{*}}{a_{2}\left(d_{2}+\beta_{2} v^{*}\right)},
\end{array}
$$

and $v^{*}$ is determined by (23).

Note that $R_{0}$ is the basic reproduction number describing the viral infection within host.

In the following, we consider the global stability of equilibria of (14).

In order to simplify the proof of the global stability of the infection equilibrium $E^{*}$, we first introduce an inequality as lemma, which was proved in [18].

Lemma 3. For $n$ positive numbers $c_{i}(i=1,2, \ldots, n)$, the inequality

$$
n-c_{1}-c_{2}-\cdots-c_{n}+\ln \left(c_{1} c_{2} \cdots c_{n}\right) \leq 0
$$

is true, and the equality holds if and only if $c_{1}=c_{2}=\cdots=c_{n}=$ 1.

Theorem 4. When $R_{0} \leq 1$, the infection-free equilibrium $E_{0}$ of system (14) is globally stable on $\Omega ;$ when $R_{0}>1$, the infection equilibrium $E^{*}$ of (14) is globally stable in the region $\Omega$.

Proof. In order to prove the global stability of the infectionfree equilibrium $E_{0}$ of (14), we define a Lyapunov function:

$$
\begin{aligned}
L_{11}= & k_{1} b_{1}\left(x_{1}-x_{10}-x_{10} \ln \frac{x_{1}}{x_{10}}\right)+k_{1} y_{1} \\
& +k_{2} b_{2}\left(x_{2}-x_{20}-x_{20} \ln \frac{x_{2}}{x_{20}}\right)+k_{2} y_{2}+v
\end{aligned}
$$


then

$$
\begin{aligned}
& \frac{d L_{11}}{d t}=k_{1} b_{1}\left(x_{1}-x_{10}\right)\left(\frac{\lambda_{1}}{x_{1}}-d_{1}-\beta_{1} v\right) \\
& +k_{1}\left[\beta_{1} b_{1} x\left(t-\tau_{1}\right) v\left(t-\tau_{1}\right)-a_{1} y_{1}\right] \\
& +k_{2} b_{2}\left(x_{2}-x_{20}\right)\left(\frac{\lambda_{2}}{x_{2}}-d_{2}-\beta_{2} v\right) \\
& +k_{2}\left[\beta_{2} b_{2} x\left(t-\tau_{2}\right) v\left(t-\tau_{2}\right)-a_{2} y_{2}\right] \\
& +k_{1} a_{1} y_{1}+k_{2} a_{2} y_{2}-\gamma v \\
& =k_{1} b_{1}\left(x_{1}-x_{10}\right)\left[\lambda_{1}\left(\frac{1}{x_{1}}-\frac{1}{x_{10}}\right)-\beta_{1} v\right] \\
& +k_{1}\left[\beta_{1} b_{1} x\left(t-\tau_{1}\right) v\left(t-\tau_{1}\right)-a_{1} y_{1}\right] \\
& +k_{2} b_{2}\left(x_{2}-x_{20}\right)\left[\lambda_{2}\left(\frac{1}{x_{2}}-\frac{1}{x_{20}}\right)-\beta_{2} v\right] \\
& +k_{2}\left[\beta_{2} b_{2} x\left(t-\tau_{2}\right) v\left(t-\tau_{2}\right)-a_{2} y_{2}\right] \\
& +k_{1} a_{1} y_{1}+k_{2} a_{2} y_{2}-\gamma v \\
& =k_{1} b_{1} \lambda_{1}\left(2-\frac{x_{1}}{x_{10}}-\frac{x_{10}}{x_{1}}\right) \\
& +k_{2} b_{2} \lambda_{2}\left(2-\frac{x_{2}}{x_{20}}-\frac{x_{20}}{x_{2}}\right) \\
& +k_{1} b_{1} \beta_{1}\left[x_{1}\left(t-\tau_{1}\right) v\left(t-\tau_{1}\right)-x_{1}(t) v(t)\right] \\
& +k_{2} b_{2} \beta_{2}\left[x_{2}\left(t-\tau_{2}\right) v\left(t-\tau_{2}\right)-x_{2}(t) v(t)\right] \\
& +\left(k_{1} b_{1} \beta_{1} x_{10}+k_{2} b_{2} \beta_{2} x_{20}-\gamma\right) v \text {. }
\end{aligned}
$$

Let $L_{1}=L_{11}+k_{1} b_{1} \beta_{1} \int_{t-\tau_{1}}^{t} x_{1}(\theta) v(\theta) d \theta+k_{2} b_{2} \beta_{2} \int_{t-\tau_{2}}^{t} x_{2}(\theta)$ $v(\theta) d \theta$; then

$$
\begin{aligned}
\frac{d L_{1}}{d t}= & k_{1} b_{1} \lambda_{1}\left(2-\frac{x_{1}}{x_{10}}-\frac{x_{10}}{x_{1}}\right) \\
& +k_{2} b_{2} \lambda_{2}\left(2-\frac{x_{2}}{x_{20}}-\frac{x_{20}}{x_{2}}\right)+\gamma\left(R_{0}-1\right) v
\end{aligned}
$$

where $R_{0}=\left(k_{1} b_{1} \beta_{1} x_{10}+k_{2} b_{2} \beta_{2} x_{20}\right) / \gamma$ is used.

It is easy to see that $2 \leq x_{i} / x_{i 0}+x_{i 0} / x_{i}(i=1,2)$, then $d L_{1} / d t \leq 0$ as $R_{0} \leq 1$. Note that, for $R_{0}<1, d L_{1} / d t=0$ if and only if $x_{i}=x_{i 0}(i=1,2)$ and $v=0$; for $R_{0}=1$, $d L_{1} / d t=0$ if and only if $x_{i}=x_{i 0}(i=1,2)$. No matter the case $R_{0}<1$ or $R_{0}=1$, the largest invariant set of (14) on the set $\Omega$ is the singleton $\left\{E_{0}\right\}$. Since any solution of (14) is bounded, it follows from the Lyapunov-LaSalle Invariance Principle for functional differential equations that the infection-free equilibrium $E_{0}$ is globally stable on the set $\Omega$ when $R_{0} \leq 1$ [19].
In order to prove the global stability of the infection equilibrium $E^{*}\left(x_{1}^{*}, y_{1}^{*}, x_{2}^{*}, y_{2}^{*}, v^{*}\right)$, define the following Lyapunov functions and functionals:

$$
\begin{gathered}
L_{21}=k_{1} b_{1}\left(x_{1}-x_{1}^{*}-x_{1}^{*} \ln \frac{x_{1}}{x_{1}^{*}}\right) \\
+k_{1}\left(y_{1}-y_{1}^{*}-y_{1}^{*} \ln \frac{y_{1}}{y_{1}^{*}}\right), \\
L_{22}=k_{2} b_{2}\left(x_{2}-x_{2}^{*}-x_{2}^{*} \ln \frac{x_{2}}{x_{2}^{*}}\right) \\
+k_{2}\left(y_{2}-y_{2}^{*}-y_{2}^{*} \ln \frac{y_{2}}{y_{2}^{*}}\right), \\
L_{24}=k_{1} b_{1} \beta_{1} x_{1}^{*} v^{*} \\
\times \int_{t-\tau_{1}}^{t}\left[\frac{x_{1}(\theta) v(\theta)}{x_{1}^{*} v^{*}}-1-\ln \frac{x_{1}(\theta) v(\theta)}{x_{1}^{*} v^{*}}\right] d \theta \\
+k_{2} b_{2} \beta_{2} x_{2}^{*} v^{*} \\
\times \int_{t-\tau_{2}}^{t}\left[\frac{x_{2}(\theta) v(\theta)}{x_{2}^{*} v^{*}}-1-\ln \frac{x_{2}(\theta) v(\theta)}{x_{2}^{*} v^{*}}\right] d \theta .
\end{gathered}
$$

According to (20), we have

$$
\begin{gathered}
d_{1}=\frac{\lambda_{1}}{x_{1}^{*}}-\beta_{1} v^{*}, \\
a_{1}=\frac{\beta_{1} b_{1} x_{1}^{*} v^{*}}{y_{1}^{*}}, \\
d_{2}=\frac{\lambda_{2}}{x_{2}^{*}-\beta_{2} v^{*},} \\
a_{2}=\frac{\beta_{2} b_{2} x_{2}^{*} v^{*}}{y_{2}^{*}}, \\
\gamma=\frac{k_{1} a_{1} y_{1}^{*}}{v^{*}}+\frac{k_{2} a_{2} y_{2}^{*}}{v^{*}} .
\end{gathered}
$$

Then, system (14) can be rewritten as

$$
\begin{gathered}
x_{1}^{\prime}(t)=x_{1}(t)\left[\lambda_{1}\left(\frac{1}{x_{1}(t)}-\frac{1}{x_{1}^{*}}\right)-\beta_{1}\left(v(t)-v^{*}\right)\right], \\
y_{1}^{\prime}(t)=\beta_{1} b_{1} y_{1}(t)\left[\frac{x_{1}\left(t-\tau_{1}\right) v\left(t-\tau_{1}\right)}{y_{1}(t)}-\frac{x_{1}^{*} v^{*}}{y_{1}^{*}}\right], \\
x_{2}^{\prime}(t)=x_{2}(t)\left[\lambda_{2}\left(\frac{1}{x_{2}(t)}-\frac{1}{x_{2}^{*}}\right)-\beta_{2}\left(v(t)-v^{*}\right)\right], \\
y_{2}^{\prime}(t)=\beta_{2} b_{2} y_{2}(t)\left[\frac{x_{2}\left(t-\tau_{2}\right) v\left(t-\tau_{2}\right)}{y_{2}(t)}-\frac{x_{2}^{*} v^{*}}{y_{2}^{*}}\right], \\
v^{\prime}(t)=v(t)\left[k_{1} a_{1}\left(\frac{y_{1}(t)}{v(t)}-\frac{y_{1}^{*}}{v^{*}}\right)+k_{2} a_{2}\left(\frac{y_{2}(t)}{v(t)}-\frac{y_{2}^{*}}{v^{*}}\right)\right] .
\end{gathered}
$$


By using $\lambda_{1}=d_{1} x_{1}^{*}+\beta_{1} x_{1}^{*} v^{*}$ and $\lambda_{2}=d_{2} x_{2}^{*}+\beta_{2} x_{2}^{*} v^{*}$, direct computations show that

$$
\begin{aligned}
& L_{21}^{\prime}=k_{1} b_{1} d_{1} x_{1}^{*}\left(2-\frac{x_{1}^{*}}{x_{1}}-\frac{x_{1}}{x_{1}^{*}}\right) \\
& +k_{1} b_{1} \beta_{1} x_{1}^{*} v^{*}\left(2-\frac{x_{1}^{*}}{x_{1}}-\frac{x_{1} v}{x_{1}^{*} v^{*}}+\frac{v}{v^{*}}\right) \\
& +k_{1} b_{1} \beta_{1} x_{1}^{*} v^{*}\left[\frac{x_{1}\left(t-\tau_{1}\right) v\left(t-\tau_{1}\right)}{x_{1}^{*} v^{*}}-\frac{y_{1}}{y_{1}^{*}}\right. \\
& \left.-\frac{x_{1}\left(t-\tau_{1}\right) y_{1}^{*} v\left(t-\tau_{1}\right)}{x_{1}^{*} y_{1} v^{*}}\right] \text {, } \\
& L_{22}^{\prime}=k_{2} b_{2} d_{2} x_{2}^{*}\left(2-\frac{x_{2}^{*}}{x_{2}}-\frac{x_{2}}{x_{2}^{*}}\right) \\
& +k_{2} b_{2} \beta_{2} x_{2}^{*} v^{*}\left(2-\frac{x_{2}^{*}}{x_{2}}-\frac{x_{2} v}{x_{2}^{*} v^{*}}+\frac{v}{v^{*}}\right) \\
& +k_{2} b_{2} \beta_{2} x_{2}^{*} v^{*}\left[\frac{x_{2}\left(t-\tau_{2}\right) v\left(t-\tau_{2}\right)}{x_{2}^{*} v^{*}}-\frac{y_{2}}{y_{2}^{*}}\right. \\
& \left.-\frac{x_{2}\left(t-\tau_{2}\right) y_{2}^{*} v\left(t-\tau_{2}\right)}{x_{2}^{*} y_{2} v^{*}}\right] \text {, } \\
& L_{23}^{\prime}=k_{1} a_{1} y_{1}^{*}\left(\frac{y_{1}}{y_{1}^{*}}-\frac{y_{1} v^{*}}{y_{1}^{*} v}-\frac{v}{v^{*}}+1\right) \\
& +k_{2} a_{2} y_{2}^{*}\left(\frac{y_{2}}{y_{2}^{*}}-\frac{y_{2} v^{*}}{y_{2}^{*} v}-\frac{v}{v^{*}}+1\right) \text {, } \\
& L_{24}^{\prime}=k_{1} b_{1} \beta_{1} x_{1}^{*} v^{*}\left[\frac{x_{1} v}{x_{1}^{*} v^{*}}-\frac{x_{1}\left(t-\tau_{1}\right) v\left(t-\tau_{1}\right)}{x_{1}^{*} v^{*}}\right. \\
& \left.+\ln \frac{x_{1}\left(t-\tau_{1}\right) v\left(t-\tau_{1}\right)}{x_{1}(t) v(t)}\right] \\
& +k_{2} b_{2} \beta_{2} x_{2}^{*} v^{*}\left[\frac{x_{2} v}{x_{2}^{*} v^{*}}-\frac{x_{2}\left(t-\tau_{2}\right) v\left(t-\tau_{2}\right)}{x_{2}^{*} v^{*}}\right. \\
& \left.+\ln \frac{x_{2}\left(t-\tau_{2}\right) v\left(t-\tau_{2}\right)}{x_{2}(t) v(t)}\right] \text {. }
\end{aligned}
$$

$$
\begin{aligned}
& \text { Let } L_{2}=L_{21}+L_{22}+L_{23}+L_{24} \text {, then } \\
& L_{2}^{\prime}=k_{1} b_{1} d_{1} x_{1}^{*}\left(2-\frac{x_{1}^{*}}{x_{1}}-\frac{x_{1}}{x_{1}^{*}}\right)+k_{2} b_{2} d_{2} x_{2}^{*}\left(2-\frac{x_{2}^{*}}{x_{2}}-\frac{x_{2}}{x_{2}^{*}}\right) \\
& +k_{1} b_{1} \beta_{1} x_{1}^{*} v^{*}\left[3-\frac{x_{1}^{*}}{x_{1}(t)}-\frac{x_{1}\left(t-\tau_{1}\right) y_{1}^{*} v\left(t-\tau_{1}\right)}{x_{1}^{*} y_{1} v^{*}}\right. \\
& \left.-\frac{y_{1}(t) v^{*}}{y_{1}^{*} v(t)}+\ln \frac{x_{1}\left(t-\tau_{1}\right) v\left(t-\tau_{1}\right)}{x_{1}(t) v(t)}\right]
\end{aligned}
$$

$$
\begin{aligned}
+k_{2} b_{2} \beta_{2} x_{2}^{*} v^{*} & {\left[3-\frac{x_{2}^{*}}{x_{2}(t)}-\frac{x_{2}\left(t-\tau_{2}\right) y_{2}^{*} v\left(t-\tau_{2}\right)}{x_{2}^{*} y_{2} v^{*}}\right.} \\
& \left.-\frac{y_{2}(t) v^{*}}{y_{2}^{*} v(t)}+\ln \frac{x_{2}\left(t-\tau_{2}\right) v\left(t-\tau_{2}\right)}{x_{2}(t) v(t)}\right] .
\end{aligned}
$$

According to the relationship between the arithmetic and the associated geometric means and Lemma $3, d L_{2} / d t \leq 0$ and $d L_{2} / d t=0$ if and only if $x_{1}=x_{1}^{*}, x_{2}=x_{2}^{*}, y_{1} / y_{1}^{*}=$ $y_{2} / y_{2}^{*}=v(t) / v^{*}=v\left(t-\tau_{1}\right) / v^{*}=v\left(t-\tau_{2}\right) / v^{*}$. It is easy to see that the largest invariant set of (14) on the set $\left\{\left(x_{1}, y_{1}, x_{2}, y_{2}, v\right) \in \Omega: d L_{2} / d t=0\right\}$ is the singleton $\left\{E^{*}\right\}$. Since any solution of (14) is bounded, it follows from the Lyapunov-LaSalle Invariance Principle for functional differential equations that the infection equilibrium $E^{*}$ is globally stable on the set $\Omega$ when $R_{0}>1$ [19].

\section{Discussion}

In this paper, we first present the probability function describing the latency of the infected cells such that some models of viral infection with latent stage are unified. When the function is an exponential one, the associated model is a system of ordinary differential equations; when the function is a step function, the associated one is a delay differential system. Both of the two types of models have the similar dynamical behaviors. That is, when the basic reproduction number is less than or equal to unity, the infection-free equilibrium is globally stable, which implies that the inhost free virus will be cleared out finally; when the basic reproduction number is greater than unity, the infection equilibrium is globally stable, which implies that the viral infection will be chronic and persist in-host $[6,7,17]$. But there is a difference between the basic reproduction numbers for the two types of models.

In fact, for ordinary differential systems (6) and (12), the basic reproduction numbers are

$$
\begin{gathered}
R_{01}^{(O)}=\frac{\beta \lambda k \varepsilon}{d \gamma(d+\varepsilon)}, \\
R_{02}^{(O)}=\left[\frac{\beta_{1} \lambda_{1} k_{1} \varepsilon_{1}}{d_{1}\left(d_{1}+\varepsilon_{1}\right)}+\frac{\beta_{2} \lambda_{2} k_{2} \varepsilon_{2}}{d_{2}\left(d_{2}+\varepsilon_{2}\right)}\right] \frac{1}{\gamma},
\end{gathered}
$$

respectively $[6,7]$. For delay differential systems (10) and (13) (or (14)), the basic reproduction numbers are

$$
\begin{gathered}
R_{01}^{(D)}=\frac{\beta \lambda k e^{-d \tau}}{d \gamma}, \\
R_{02}^{(D)}=\left[\frac{\beta_{1} \lambda_{1} k_{1} e^{-d_{1} \tau_{1}}}{d_{1}}+\frac{\beta_{2} \lambda_{2} k_{2} e^{-d_{2} \tau_{2}}}{d_{2}}\right] \frac{1}{\gamma},
\end{gathered}
$$

respectively, where $R_{01}^{(D)}$ was obtained in [17]; $R_{02}^{(D)}$ is $R_{0}$ in this paper.

According to the definition of probability function of staying in the latent stage, for the two common types of probability functions, exponential function and step function, we 
assume that the associated average latent periods of infected cells are equal, that is, $\tau=1 / \varepsilon$. Then, the basic reproduction numbers of ordinary differential systems (6) and (12) can be rewritten by

$$
\begin{gathered}
R_{01}^{(O)}=\frac{\beta \lambda k}{d \gamma(1+d \tau)}, \\
R_{02}^{(O)}=\left[\frac{\beta_{1} \lambda_{1} k_{1}}{d_{1}\left(1+d_{1} \tau_{1}\right)}+\frac{\beta_{2} \lambda_{2} k_{2}}{d_{2}\left(1+d_{2} \tau_{2}\right)}\right] \frac{1}{\gamma},
\end{gathered}
$$

respectively. that

From the inequality $1 /(1+u)>e^{-u}$ for $u>0$, it follows

$$
R_{01}^{(O)}>R_{01}^{(D)}, \quad R_{02}^{(O)}>R_{02}^{(D)} .
$$

They imply that for certain models of viral infection, the basic reproduction number of ordinary differential system may be greater than that of delay differential system. It is well known that the basic reproduction number usually determines dynamics of models of viral infection. Therefore, the difference between the basic reproduction numbers of the different types of models reminds us that it is necessary to elect an appropriate type of probability function, when predicting the final outcome of viral infection in-host.

\section{Acknowledgments}

The authors would like to thank the referees for their valuable comments. This work was partially supported by the National Natural Science Foundation of China (nos. 11071256, 11171267, and 11371369).

\section{References}

[1] M. A. Nowak, S. Bonhoeffer, A. M. Hill, R. Boehme, H. C. Thomas, and H. McDade, "Viral dynamics in hepatitis B virus infection," Proceedings of the National Academy of Sciences of the United States of America, vol. 93, no. 9, pp. 4398-4402, 1996.

[2] M. A. Nowak and R. M. May, Virus Dynamics: Mathematical Principles of Immunology and Virology, Oxford University Press, 2000.

[3] D. D. Ho, R. J. Pomerantz, and J. C. Kaplan, "Pathogenesis of infection with human immunodeficiency virus," The New England Journal of Medicine, vol. 317, no. 5, pp. 278-286, 1987.

[4] A. S. Perelson, D. E. Kirschner, and R. D. DeBoer, "Dynamics of HIV infection of $\mathrm{CD}^{+}{ }^{+} \mathrm{T}$ cells," Mathematical Biosciences, vol. 114, no. 1, pp. 81-125, 1993.

[5] A. S. Perelson and P. W. Nelson, "Mathematical analysis of HIV1 dynamics in vivo," SIAM Review, vol. 41, no. 1, pp. 3-44, 1999.

[6] A. Korobeinikov, "Global properties of basic virus dynamics models," Bulletin of Mathematical Biology, vol. 66, no. 4, pp. 879-883, 2004.

[7] A. M. Elaiw, "Global properties of a class of HIV models," Nonlinear Analysis: Real World Applications, vol. 11, no. 4, pp. 2253-2263, 2010.

[8] A. S. Perelson, P. Essunger, Y. Cao et al., "Decay characteristics of HIV-1-infected compartments during combination therapy," Nature, vol. 387, no. 6629, pp. 188-191, 1997.
[9] D. Sansonno, A. R. Iacobelli, V. Cornacchiulo, G. Iodice, and F. Dammacco, "Detection of hepatitis $\mathrm{C}$ virus (HCV) proteins by immunofluorescence and HCV RNA genomic sequences by non-isotopic in situ hybridization in bone marrow and peripheral blood mononuclear cells of chronically HCV-infected patients," Clinical and Experimental Immunology, vol. 103, no. 3, pp. 414-421, 1996.

[10] H. Lerat, S. Rumin, F. Habersetzer et al., "In vivo tropism of hepatitis $\mathrm{C}$ virus genomic sequences in hematopoietic cells: influence of viral load, viral genotype, and cell phenotype," Blood, vol. 91, no. 10, pp. 3841-3849, 1998.

[11] F. Maggi, M. Giorgi, C. Fornai et al., "Detection and quasispecies analysis of hepatitis $\mathrm{C}$ virus in the cerebrospinal fluid of infected patients," Journal of NeuroVirology, vol. 5, no. 3, pp. 319-323, 1999.

[12] S. Pal, D. G. Sullivan, S. Kim et al., "Productive replication of hepatitis $\mathrm{C}$ virus in perihepatic lymph nodes in vivo: implications of HCV lymphotropism," Gastroenterology, vol. 130, no. 4, pp. 1107-1116, 2006.

[13] D. S. Callaway and A. S. Perelson, "HIV-1 infection and low steady state viral loads," Bulletin of Mathematical Biology, vol. 64, no. 1, pp. 29-64, 2002.

[14] R. Qesmi, J. Wu, J. Wu, and J. M. Heffernan, "Influence of backward bifurcation in a model of hepatitis B and C viruses," Mathematical Biosciences, vol. 224, no. 2, pp. 118-125, 2010.

[15] H. W. Hethcote, H. W. Stech, and P. van den Driessche, "Stability analysis for models of diseases without immunity," Journal of Mathematical Biology, vol. 13, no. 2, pp. 185-198, 1981.

[16] H. W. Hethcote and P. van den Driessche, "Two SIS epidemiologic models with delays," Journal of Mathematical Biology, vol. 40, no. 1, pp. 3-26, 2000.

[17] M. Y. Li and H. Shu, "Global dynamics of an in-host viral model with intracellular delay," Bulletin of Mathematical Biology, vol. 72, no. 6, pp. 1492-1505, 2010.

[18] J. Li, X. Song, and F. Gao, "Global stability of a viral infection model with two delays and two types of target cells," The Journal of Applied Analysis and Computation, vol. 2, no. 3, pp. 281-292, 2012.

[19] J. Hale and S. M. Verduyn Lunel, Introduction to Functional Differential Equations, vol. 99 of Applied Mathematical Science, New York, NY, USA, 1993. 


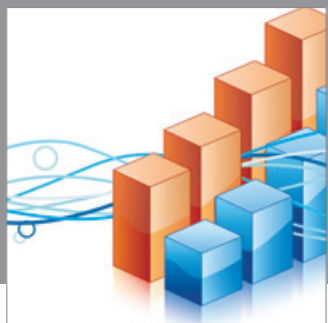

Advances in

Operations Research

mansans

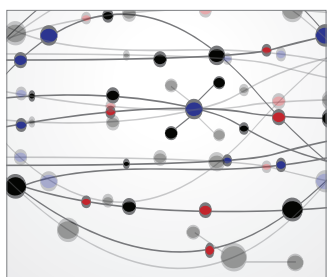

The Scientific World Journal
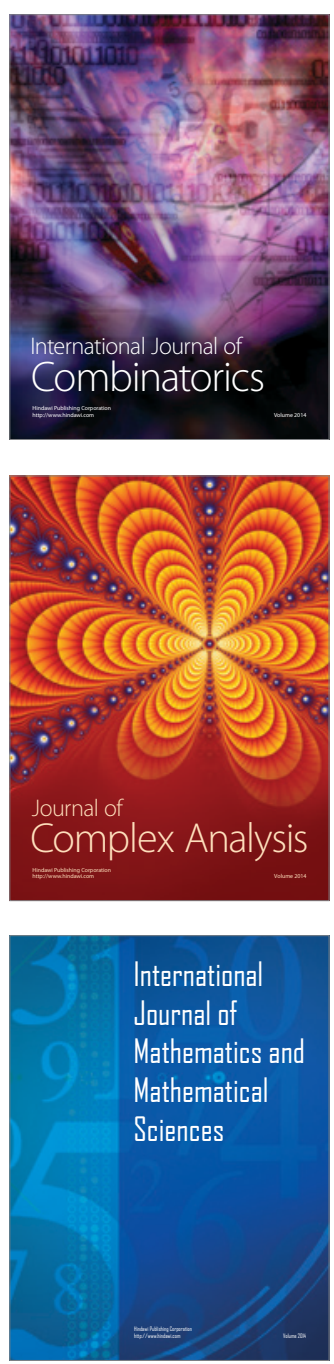
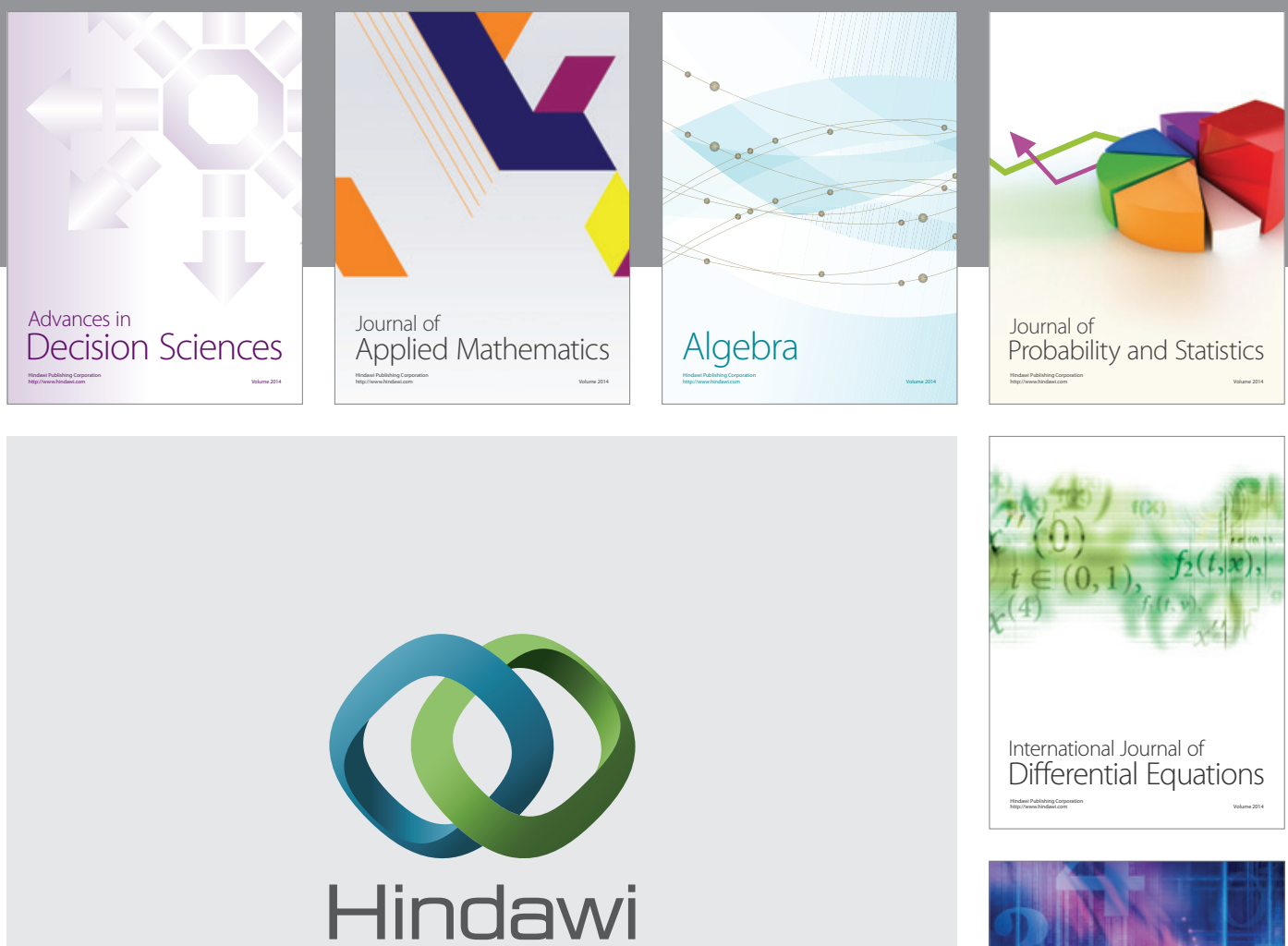

Submit your manuscripts at http://www.hindawi.com
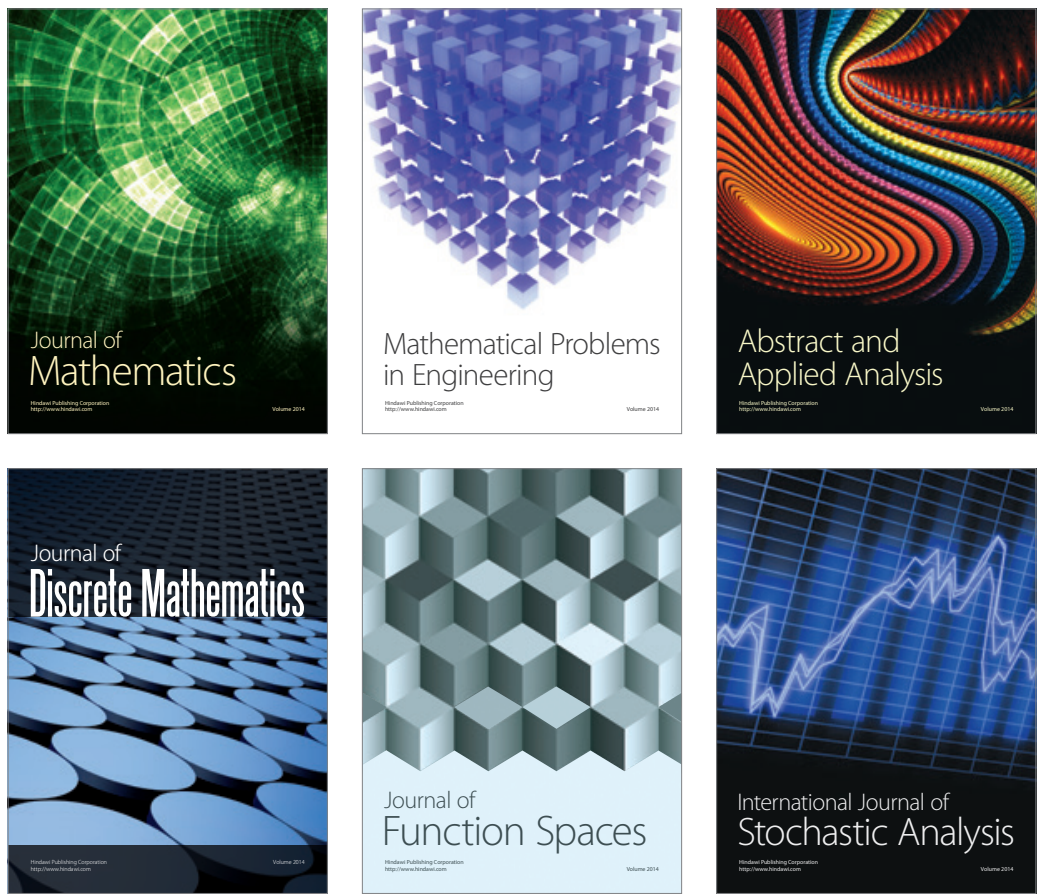

Journal of

Function Spaces

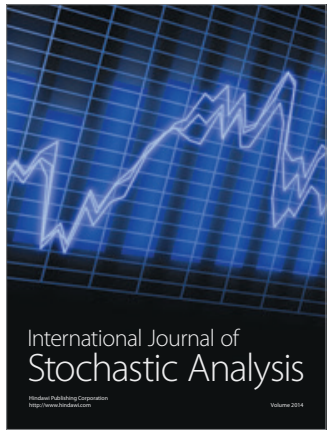

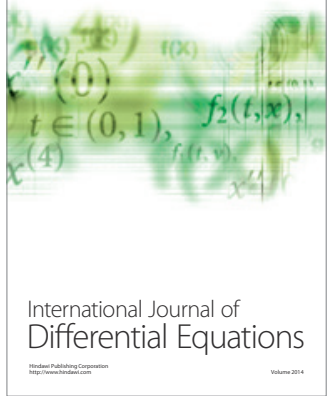
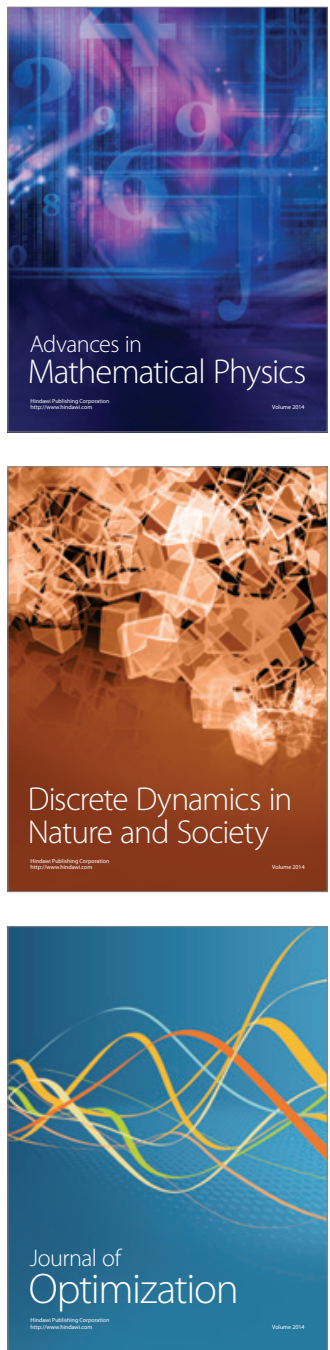